\title{
Triggered Electrooculography for Identification of Oculomo- tor and Abducens Nerves during Skull Base Surgery
}

\author{
Ha-Neul Jeong, ${ }^{1,2}$ Sang-Il Ahn, ${ }^{1}$ Minkyun Na, Jihwan Yoo, ${ }^{3}$ Woohyun Kim, ${ }^{3}$ In-Ho Jung, ${ }^{3}$ Soobin Kang, ${ }^{1}$ Seung Min Kim, \\ Ha Young Shin, Jong Hee Chang, ${ }^{3,4}$ Eui Hyun Kim ${ }^{3,4}$ \\ Department of Neurology, ${ }^{\top}$ Yonsei University College of Medicine, Seoul, Korea \\ Department of Neurology, ${ }^{2}$ Myongji Hospital, Goyang, Korea \\ Department of Neurosurgery, ${ }^{3}$ Yonsei University College of Medicine, Seoul, Korea \\ Brain Tumor Center, ${ }^{4}$ Severance Hospital, Seoul, Korea
}

Objective : Electrooculography (EOG) records eyeball movements as changes in the potential difference between the negatively charged retina and the positively charged cornea. We aimed to investigate whether reliable EOG waveforms can be evoked by electrical stimulation of the oculomotor and abducens nerves during skull base surgery.

Methods : We retrospectively reviewed the records of 18 patients who had undergone a skull base tumor surgery using EOG (11 craniotomies and seven endonasal endoscopic surgeries). Stimulation was performed at $5 \mathrm{~Hz}$ with a stimulus duration of $200 \mu \mathrm{s}$ and an intensity of $0.1-5 \mathrm{~mA}$ using a concentric bipolar probe. Recording electrodes were placed on the upper (active) and lower (reference) eyelids, and on the outer corners of both eyes; the active electrode was placed on the contralateral side.

Results : Reproducibly triggered EOG waveforms were observed in all cases. Electrical stimulation of cranial nerves (CNs) III and VI elicited positive waveforms and negative waveforms, respectively, in the horizontal recording. The median latencies were 3.1 and $0.5 \mathrm{~ms}$ for craniotomies and endonasal endoscopic surgeries, respectively $(p=0.007)$. Additionally, the median amplitudes were 33.7 and $46.4 \mu \mathrm{V}$ for craniotomies and endonasal endoscopic surgeries, respectively $(p=0.40)$.

Conclusion : This study showed reliably triggered EOG waveforms with stimulation of CNs III and VI during skull base surgery. The latency was different according to the point of stimulation and thus predictable. As EOG is noninvasive and relatively easy to perform, it can be used to identify the ocular motor nerves during surgeries as an alternative of electromyography.

Key Words : Abducens nerve · Electrooculography · Monitoring, Intraoperative · Oculomotor nerve.

\section{INTRODUCTION}

Intraoperative neurophysiologic monitoring (IONM) of extraocular cranial nerves $(\mathrm{CNs})$ is useful in surgeries involving the skull base, brainstem, cavernous sinus, and orbital region.
In many previous studies, direct electromyography was used to monitor the ocular motor nerve function to prevent postoperative neurologic deficits ${ }^{3,8)}$. Nevertheless, IONM of ocular motor function is not commonly performed because of technical difficulties in placing the recording electrodes into the

- Received : June 22, 2020 •Revised : July 31, 2020 •Accepted : August 10, 2020

- Address for reprints : Eui Hyun Kim

Department of Neurosurgery, Yonsei University College of Medicine, 50-1 Yonsei-ro, Seodaemun-gu, Seoul 03722, Korea

Tel : +82-2-2228-2150, Fax : +82-2-393-9979, E-mail : euihyunkim@yuhs.ac, ORCID : https://orcid.org/0000-0002-2523-7122

This is an Open Access article distributed under the terms of the Creative Commons Attribution Non-Commercial License (http://creativecommons.org/licenses/by-nc/4.0) which permits unrestricted non-commercial use, distribution, and reproduction in any medium, provided the original work is properly cited. 
extraocular muscles. As an alternative to this approach, electrooculography (EOG) recording of eyeball movements was used to detect changes in the potential difference between the negatively charged retina and the positively charged cornea ${ }^{2)}$. Very few researchers ${ }^{2,7,11)}$ suggested the usefulness of EOG for monitoring ocular motor function, however, there is no consensus on the use of a particular method to date. Thus, the aim of this study was to investigate whether reliable EOG waveforms can be evoked by electrical stimulation of the oculomotor and abducens nerves during skull base surgery.

\section{MATERIALS AND METHODS}

The study design was approved by the Appropriate Ethics Review Board of Severance Hospital (4-2019-0500).

\section{Patients}

The records of 18 patients who had undergone brain tumor surgery were examined. The types of surgical approaches used were endonasal endoscopic surgeries (seven patients) and craniotomy (11 patients). In endonasal endoscopic cases, all tu-

Table 1. Demographic and clinical information of patients

\begin{tabular}{|c|c|c|}
\hline & $\begin{array}{l}\text { Patients with } \\
\text { craniotomy }\end{array}$ & $\begin{array}{c}\text { Patients with } \\
\text { endonasal } \\
\text { endoscopic surgery }\end{array}$ \\
\hline Total number & 11 & 7 \\
\hline Sex, F:M & $11: 0$ & $5: 2$ \\
\hline Age (years) & $57.7 \pm 9.1$ & $51.0 \pm 19.4$ \\
\hline \multicolumn{3}{|l|}{ Pathologic diagnosis } \\
\hline Pituitary adenoma & 3 & 2 \\
\hline Meningioma & 6 & - \\
\hline Schwannoma & 2 & 2 \\
\hline Craniopharyngioma & - & 1 \\
\hline Hemangiopericytoma & - & 1 \\
\hline Angioleiomyoma & - & 1 \\
\hline \multicolumn{3}{|l|}{ Clinical outcome } \\
\hline Preoperative CN III palsy & - & 1 \\
\hline Preoperative CN VI palsy & 1 & - \\
\hline Postoperative CN III palsy & 2 & - \\
\hline Postoperative CN VI palsy & - & - \\
\hline
\end{tabular}

Values are presented as mean \pm standard deviation or number unless otherwise indicated. $\mathrm{F}$ : female, $\mathrm{M}$ : male, CN : cranial nerve mors were encroaching or adjoining the oculomotor and/or abducens nerve as they were directly invading or apparently compressing cavernous sinuses or orbits. Monitoring indications were skull base lesions of diverse origins : meningioma (six patients), pituitary adenoma (five patients), schwannoma (four patients), craniopharyngioma (one patient), hemangiopericytoma (one patient), and angioleiomyoma (one patient) (Table 1). All patients received intravenous anesthesia with propofol and remifentanil. Orotracheal intubation was facilitated by the use of rocuronium; thereafter, no additional muscle relaxants were administered.

\section{Intraoperative EOG}

The Eclipse neuromonitoring system (Medtronic Xomed Inc., Jacksonville, FL, USA) was used to trigger EOG waveforms. A 100-mm-long Medtronic concentric bipolar probe was used. Stimulation was performed at $5 \mathrm{~Hz}$ with a stimulus duration of $200 \mu$ s and an intensity of $0.1-5 \mathrm{~mA}$ until a response was observed. The surgeon was able to identify and stimulate the oculomotor and abducens nerves under direct visualization during surgery. During endonasal endoscopic surgery, the abducens and oculomotor nerves were indirectly stimulated while tumors were not removed.

Two channels were recorded using surface electrodes. One pair of electrodes was placed on the upper (active) and lower (reference) eyelids of the examined eye for the vertical recording. The other pair of electrodes was placed on the outer corners of both eyes for the horizontal recording; the active and reference electrodes were placed on the contralateral and ipsilateral sides, respectively (Fig. 1). The ground electrode was placed on the proximal upper extremity. The electrical condi-

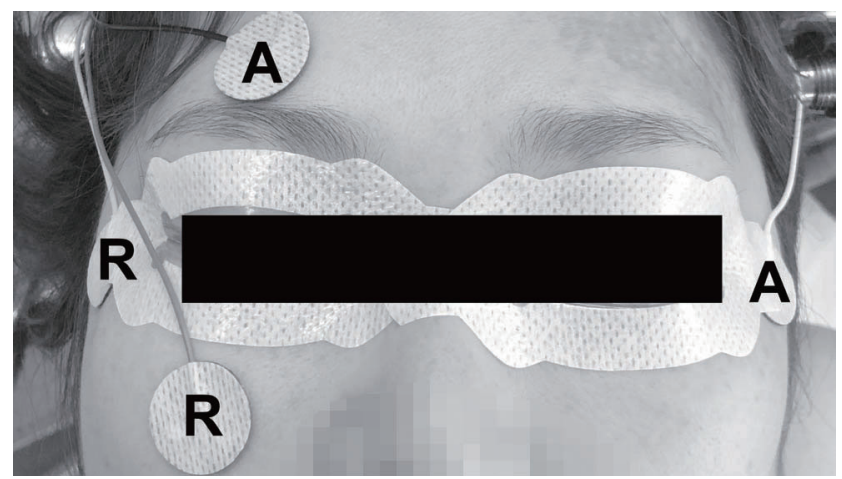

Fig. 1. A schematic design showing the placement of electrodes for the intraoperative electrooculography. $R$ : reference electrode, $A$ : active electrode. 
tions for measurements were the following : sensitivity, 50 $\mu \mathrm{V} / \mathrm{div}$; time base, $1 \mathrm{~ms} / \mathrm{div}$; low-frequency filter, $10 \mathrm{~Hz}$; and high-frequency filter, $1 \mathrm{kHz}$.

The responses were interpreted by neurophysiologic technicians accompanied by a supervising neurophysiologist in the operating room. The morphology, amplitude, and latency of each triggered EOG waveform were analyzed. Amplitudes were measured using the peak-to-peak method, and latencies were measured as the distance to the initial peak. The difference in latency and amplitude of EOG waveforms between craniotomy and endoscopic endonasal surgery was evaluated by t-test. $p$ - value $<0.05$ was considered statistically significant.

\section{RESULTS}

\section{Triggered EOG waveforms}

Triggered EOG waveforms were observed in all 18 cases. CN III was examined in 12 patients and CN VI was examined in 10 patients. Fourteen waveforms were obtained during the craniotomies, and eight waveforms were obtained during the endonasal endoscopic surgeries; eventually, 22 EOG wave-

Table 2. Characteristics of the triggered electrooculographic waveforms in 36 patients

\begin{tabular}{|c|c|c|}
\hline & Craniotomy & Endoscopic endonasal surgery \\
\hline Total number of observed waveforms & 14 & 8 \\
\hline \multicolumn{3}{|l|}{ Numbers of cases with examined nerves } \\
\hline Cranial nerve III & 7 & 5 \\
\hline Cranial nerve VI & 7 & 3 \\
\hline \multicolumn{3}{|c|}{$\begin{array}{l}\text { Threshold of stimulation for triggered electrooculographic waveforms } \\
(\mathrm{mA})\end{array}$} \\
\hline $0.1-0.4$ & 4 & - \\
\hline 0.5 & 3 & 5 \\
\hline 1 & 5 & 3 \\
\hline 2 & 2 & - \\
\hline \multicolumn{3}{|l|}{ Morphology of waveform } \\
\hline Biphasic & 8 & 8 \\
\hline Monophasic & 6 & - \\
\hline Amplitude of waveform $(\mu \mathrm{V})$ & $33.7(26.0-71.8)$ & $46.4(32.1-55.7)$ \\
\hline Latency of waveform (ms) & $3.1(0.5-3.4)$ & $0.5(0.4-0.5)$ \\
\hline
\end{tabular}

Values are presented as median (interquartile range) or number
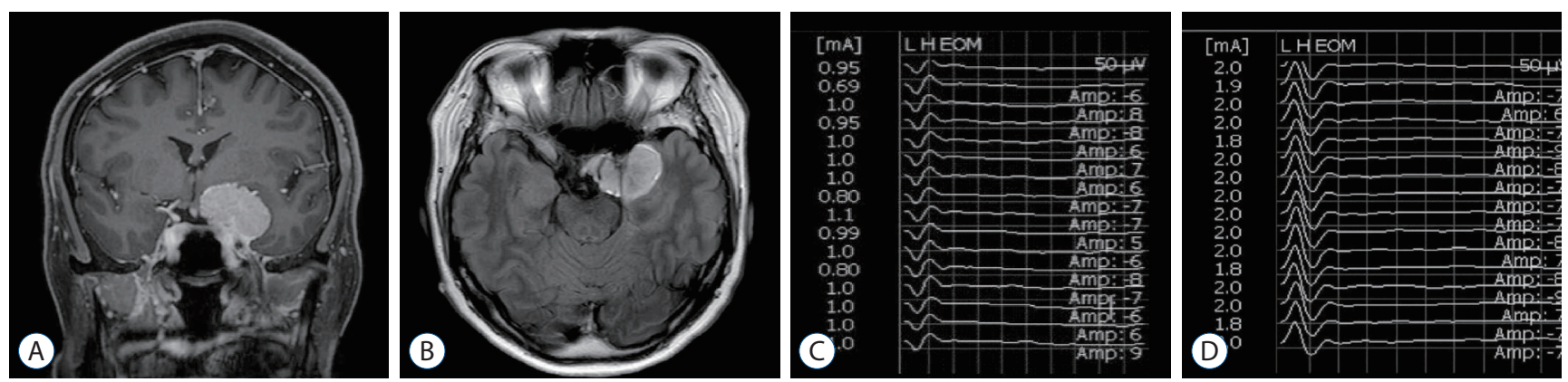

Fig. 2. T1-weighted coronal (A) and fluid attenuated inversion recovery axial (B) magnetic resonance images with enhancement showed left anterior clinoid process meningioma. The triggered electrooculographic responses of left eye in horizontal recording showed the biphasic positive waveforms with a latency of 0.5 $\mathrm{ms}$ and an amplitude of $50 \mu \mathrm{V}$ when stimulating left oculomotor nerve at a stimulation intensity of $1 \mathrm{~mA}(\mathrm{C})$ and the biphasic negative waveforms with a latency of $0.5 \mathrm{~ms}$ and an amplitude of $75 \mu \mathrm{V}$ when stimulating left abducens nerve at a stimulation intensity of $2 \mathrm{~mA}$ (D). 
forms were analyzed (Table 2).

The responses in the vertical traces were either not observed or inconsistent, regardless of the specific nerve stimulated, and the responses in the horizontal traces were consistently observed when each nerve was stimulated. Electrical stimulation of CN III elicited positive peak, indicating movements of the eyeball toward the active electrode. Electrical stimulation of CN VI elicited negative peak, indicating movements of the eyeball away from the active electrode (Fig. 2).

Reproducible EOG waveforms were triggered at an intensity of less than $0.5 \mathrm{~mA}$ in $50 \%$ of cases (7/14) of craniotomies and $62.5 \%$ of cases (5/8) of endonasal endoscopic surgeries). Two types of EOG waveforms were observed in the craniotomy cases: One type had a latency of 3-4 ms, a long duration, and a monophasic morphology (case 1), and the other had a latency of $0.5 \mathrm{~ms}$, a short duration, and a biphasic morphology (case 2). The waveforms observed in the endonasal endoscopic surgeries were all similar, with a latency of $0.5 \mathrm{~ms}$, a short duration, and a biphasic morphology (case 3). The median amplitudes were 33.7 and $46.4 \mu \mathrm{V}$ for craniotomies and endonasal endoscopic surgeries, respectively $(p=0.40)$. However, the median latencies were 3.1 and $0.5 \mathrm{~ms}$ for craniotomies and endonasal endoscopic surgeries, respectively $(p=0.007)$. The results of all cases are presented in the supplementary tables (Supplementary Tables 1 and 2).

\section{Clinical outcomes}

One patient who had undergone endonasal endoscopic surgery for a craniopharyngioma had preoperative oculomotor dysfunction prior to surgery, which resolved after surgery (case 3). One patient who had undergone craniotomy for a trigeminal schwannoma had preoperative abducens dysfunction. Two patients who underwent craniotomy for pituitary adenomas experienced postoperative oculomotor dysfunction. There were no specific differences in the waveforms of these four patients with regard to ocular motor dysfunction.

\section{Illustrative cases}

\section{Case 1}

A 69-year-old woman was admitted with a left petrous meningioma and complained of a headache. She underwent a craniotomy with tumor removal. The neurosurgeon identified and stimulated the abducens nerve during the tumor resection. We observed negative monophasic waveforms with a latency of $3.2 \mathrm{~ms}$ and an amplitude of $27 \mu \mathrm{V}$ at a stimulation intensity of $0.2 \mathrm{~mA}$ in the horizontal recording (Fig. 3). She had no postoperative ocular motor dysfunction.

\section{Case 2}

A 57-year-old woman was admitted with a left anterior clinoid process meningioma that had been incidentally found. She underwent a craniotomy with tumor removal. The neurosurgeon identified and stimulated the oculomotor and abducens nerves during the tumor resection. We observed biphasic waveforms with a latency of $0.5 \mathrm{~ms}$ in the horizontal recording. Positive waveforms with an amplitude of $50 \mu \mathrm{V}$ were observed when the oculomotor nerve was stimulated at an intensity of $1 \mathrm{~mA}$, and negative waveforms with an amplitude of $75 \mu \mathrm{V}$ were observed when the abducens nerve was stimulated
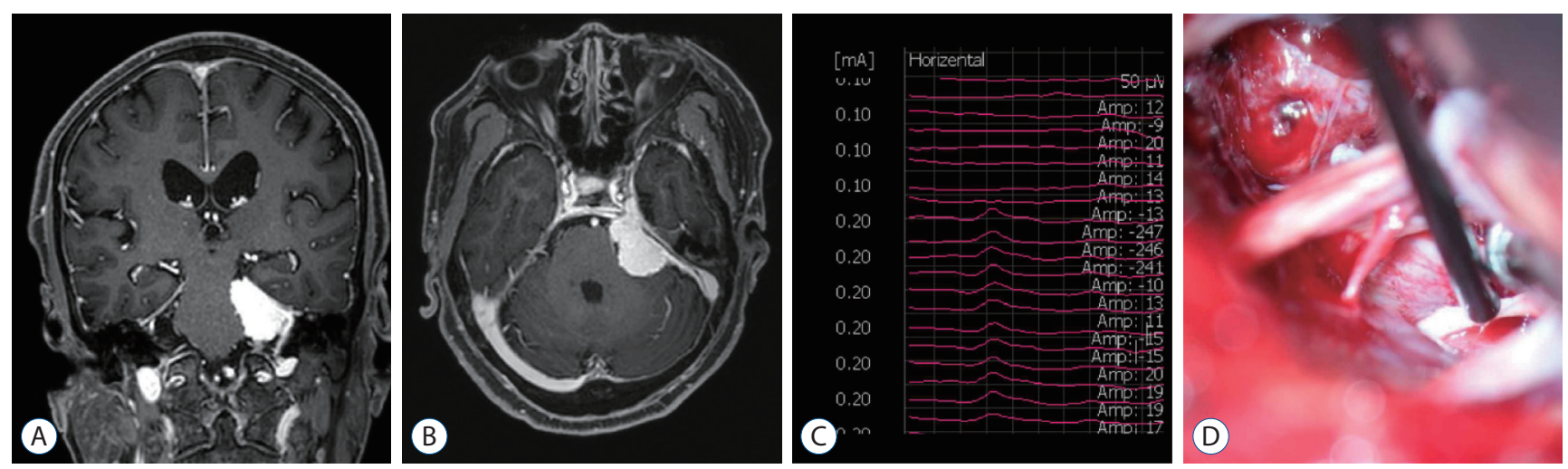

Fig. 3. T1-weighted coronal (A) and axial (B) magnetic resonance images with enhancement showed left petrous meningioma. The triggered electrooculographic responses of left eye in horizontal recording showed the monophasic negative waveforms with a latency of $3.2 \mathrm{~ms}$ and an amplitude of $27 \mu \mathrm{V}(\mathrm{C})$ when stimulating left abducens nerve at a stimulation intensity of $0.2 \mathrm{~mA}(\mathrm{D})$. 
at an intensity of $2 \mathrm{~mA}$ (Fig. 2). She had no postoperative ocular motor dysfunction.

\section{Case 3}

A 22-year-old woman was admitted with recurrent craniopharyngioma in the right sellar area. Although she had undergone craniotomy and resection of the craniopharyngioma twice before, she had relapsing progressive right CN III palsy. Thus, she underwent removal of the recurrent sellar tumor via endonasal endoscopic surgery. The neurosurgeon stimulated the oculomotor nerve indirectly over the dural layer during the tumor resection (Fig. 4). We observed positive biphasic waveforms with a latency of $0.6 \mathrm{~ms}$ and an amplitude of 61.1 $\mu \mathrm{V}$ at a stimulation intensity of $1 \mathrm{~mA}$ in the horizontal recording. One month after surgery, her right CN III palsy improved, and she had neither diplopia nor ptosis.

\section{DISCUSSION}

There have been few reports of the use of EOG to monitor the ocular motor nerves ${ }^{2,7,11}$. Kawamata et al. ${ }^{7)}$ used free-run EOG to track the ocular motor nerves during endoscopic endonasal surgery and showed that 100 cases of free-run EOG monitoring prevented postoperative diplopia compared to cases without monitoring. This technique records a waveform generated by mechanical stimulation of ocular motor nerves by surgical manipulation. However, for detailed localization of ocular motor nerves in a distorted anatomical orientation caused by tumors, triggered EOG has a distinguished value as reported in the literature ${ }^{2,11)}$. Similarly, we showed that reproducible EOG waveforms were triggered in the horizontal recording when the oculomotor and abducens nerves were stimulated during skull base surgery. We used bipolar instead of monopolar stimulation, which has been used in previous studies $^{2,11)}$, because we believe that bipolar stimulation with a focal application of current ${ }^{12)}$ is more suitable for the verification of stimulus-induced potentials when the nerve is identified. The most important observation was the difference in latency of ocular motor nerves between craniotomies and endoscopic endonasal surgery. As the stimulation sites in cavernous sinus and orbit were much closer from extraocular muscles, the latency was much shorter in patients with endoscopic endonasal surgery. On the contrary, we did not witness significant difference in the amplitude of waveforms between two groups. Focusing on the five invasive pituitary adenoma cases (three craniotomies and two endoscopic endonasal surgeries), the latency was very different between two groups. The latency of waveforms in three craniotomy cases was much longer than in two endoscopic endonasal cases and even similar with that of posterior fossa tumor patients (Supplementary Tables 1 and 2). In three craniotomy cases, the tumors inside cavernous sinus and in the cisternal space were large enough to compress and even stretch ocular motor nerves which might be the reason for the increased latency.

In our study, most waveforms showed a biphasic morphology, in contrast to the monophasic waveforms observed in previous reports ${ }^{2,11)}$. When ocular nerves were directly stimulated,
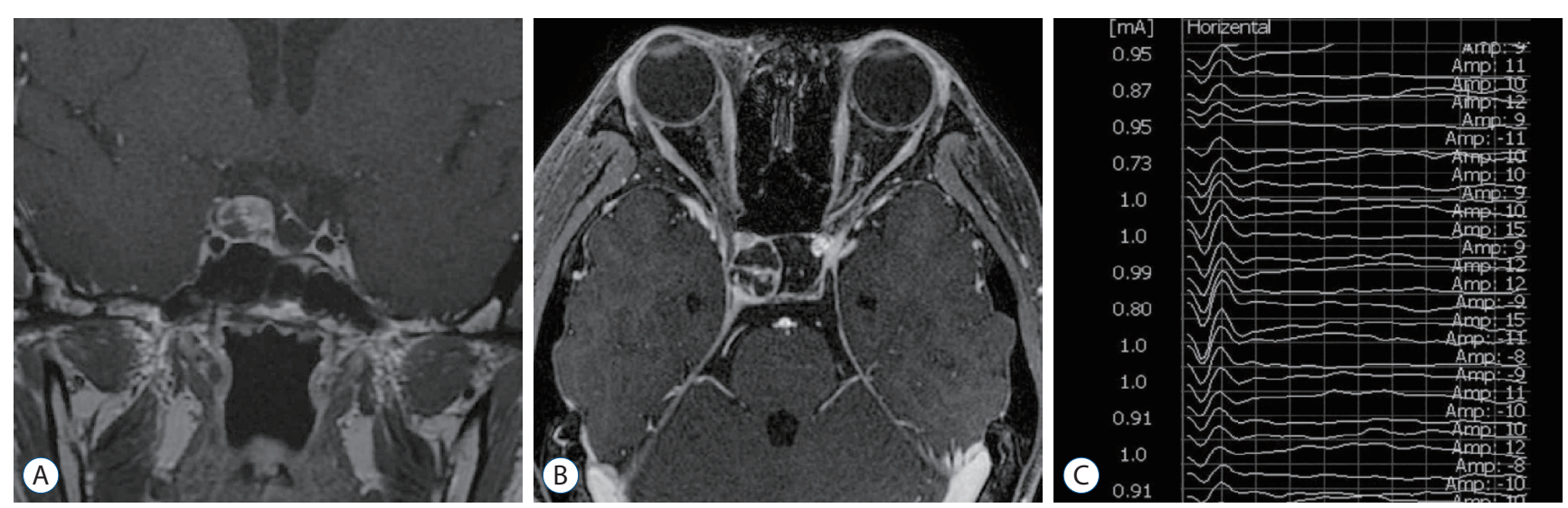

Fig. 4. T1-weighted coronal (A) and axial (B) magnetic resonance images with enhancement showed recurrent craniopharyngioma in right sellar area. The triggered electrooculographic responses of right eye in horizontal recording showed the biphasic positive waveforms with a latency of $0.6 \mathrm{~ms}$ and an amplitude of $61.1 \mu \mathrm{V}(\mathrm{C})$ when stimulating right oculomotor nerve indirectly over the dural layer at a stimulation intensity of $1 \mathrm{~mA}$. 
the waveforms were obtained at a stimulus intensity of less than $1 \mathrm{~mA}$ during either craniotomy or endoscopic endonasal surgery. We have not found any correlation between the morphology of the waveform and clinical outcome. It is possible that because the nerve stimulation site is distant from the point of injury, the functional integrity of the nerve is not reflected in the triggered EOG responses. Additionally, we triggered EOG responses only once during surgery to validate the method but did not continuously track either the free-run EOG or the changes in triggered EOG waveforms during surgery. Thus, we could not monitor in real time whether there was nerve damage during surgery.

At present, the conventional method for intraoperative ocular motor nerve monitoring involves electromyography of the extraocular muscles. The extraocular motor units have an innervation ratio of approximately three, the motor units are tightly packed, and the recording electrodes should be placed close to the neural generators to optimize the signal-to-noise ratio $^{3)}$. Schlake et al. ${ }^{9)}$ used a single-shafted bipolar needle electrode with an orbital ultrasound device to improve safety and accuracy during the insertion of the recording electrodes. Free-run electromyography is used for real-time monitoring of neural structure injuries, and evoked compound muscle action potential (CMAP) is used to map nerve trajectories or brainstem nuclei. Parameters that predict clinical outcomes include abnormal spontaneous activity in free-run electromyography, CMAP amplitude, and stimulus threshold ${ }^{6,9,10)}$. Abnormal responses in electromyography, such as neurotonic discharges, suggest axonal injury, but correlations with postoperative ocular dysfunction are poor ${ }^{9,13}$. In contrast, evoked CMAP has been used to identify neural structures during cavernous meningioma surgeries, and comparisons of basal and tracking CMAP procedures are unable to predict clinical outcomes ${ }^{4)}$. Zhou et al. ${ }^{14)}$ also showed that the evoked CMAP amplitude was the most significant parameter for predictions of oculomotor nerve function during the clipping of posterior communicating aneurysms. However, there is no consensus on which parameters should be closely controlled during intraoperative ocular motor nerve monitoring with electromyography to improve clinical outcomes.

Postoperative ocular motor dysfunction is a serious complication that can lead to diplopia, secondary amblyopia, and even functional blindness. Therefore, IONM of the ocular motor nerve is necessary to prevent such deficits, although the reported rates of injury are relatively low ${ }^{5}$. EOG is noninvasive and relatively easy to perform, so it can be used in routine clinical practice. This study showed that triggered EOG waveforms during skull base surgery can be used reliably to localize the ocular motor nerves as an alternative of electromyography. In all cases, we identified consistent and reliable EOG waveforms that correlated relevant ocular motor nerves very well. EOG can also help identify neural structures, particularly when tumors distort normal anatomic relationships ${ }^{11}$. Since monopolar stimulation is preferred to identify the nerves engulfed by tumors, a comparative study is needed to determine which type of stimulation is more appropriate for mapping. Free-run EOG monitoring must also be performed. It is not only necessary to monitor responses in real time but also to verify that a basal orbit position may not be neutral during anesthesia. Additionally, tracking triggered EOG waveforms may help predict patient prognosis by monitoring the functional integrity of the ocular motor nerve. Further studies with a larger number of patients are needed to evaluate the benefits of this approach on clinical outcomes.

\section{CONCLUSION}

We examined triggered EOG responses in 18 cases of skull base tumor surgery and found that reproducible waveforms were obtained in all cases. The latency was different according to the point of stimulation and thus predictable. As EOG is noninvasive and relatively easy to perform, it can be used to identify the ocular motor nerves during surgeries as an alternative of electromyography.

\section{CONFLICTS OF INTEREST}

No potential conflict of interest relevant to this article was reported.

\section{INFORMED CONSENT}

Informed consent was obtained from all individual participants included in this study. 


\section{AUTHOR CONTRIBUTIONS}

\author{
Conceptualization : HNJ, EHK \\ Data curation : HNJ, SIA, SK \\ Formal analysis: $\mathrm{HNJ}$, EHK \\ Methodology : HNJ, EHK \\ Project administration : $\mathrm{HNJ}$ \\ Visualization : HNJ, MN, JY, WK, IHJ \\ Writing - original draft : HNJ \\ Writing - review \& editing : SMK, HYS, JHC, EHK
}

\section{ORCID}

$\begin{array}{ll}\text { Ha-Neul Jeong } & \text { https://orcid.org/0000-0002-5785-116X } \\ \text { Sang-Il Ahn } & \text { https://orcid.org/0000-0002-2416-6174 } \\ \text { Minkyun Na } & \text { https://orcid.org/0000-0001-6826-8490 } \\ \text { Jihwan Yoo } & \text { https://orcid.org/0000-0001-8746-1245 } \\ \text { Woohyun Kim } & \text { https://orcid.org/0000-0002-2936-3740 } \\ \text { In-Ho Jung } & \text { https://orcid.org/0000-0002-4135-5743 } \\ \text { Soobin Kang } & \text { https://orcid.org/0000-0003-1214-2770 } \\ \text { Seung Min Kim } & \text { https://orcid.org/0000-0002-4384-9640 } \\ \text { Ha Young Shin } & \text { https://orcid.org/0000-0002-4408-8265 } \\ \text { Jong Hee Chang } & \text { https://orcid.org/0000-0003-1509-9800 } \\ \text { Eui Hyun Kim } & \text { https://orcid.org/0000-0002-2523-7122 }\end{array}$

\section{- Acknowledgements}

This study was funded in part by the Basic Science Research Program through the NRF of Korea (NRF-2018R1C1B5042687) funded by the Korean Ministry of Science, ICT and Future Planning (Eui Hyun Kim).

This work was presented at the 7th Congress of the international society of intraoperative neurophysiology, Vienna, Austria (October 31 to November 2, 2019).

\section{- Supplementary materials}

The online-only data supplement is available with this article at https://doi.org/10.3340/jkns.2020.0179.

\section{References}

1. Cornelius JF, Schipper J, Tortora A, Krause-Molle Z, Smuga M, Petridis $A K$, et al. : Continuous and dynamic facial nerve mapping during surgery of cerebellopontine angle tumors: clinical pilot series. World Neurosurg 119 : e855-e863, 2018

2. Fukaya C, Katayama Y, Kasai M, Kurihara J, Yamamoto T : Intraoperative electrooculographic monitoring of oculomotor nerve function during skull base surgery. Technical note. J Neurosurg 91 : 157-159, 1999

3. Hariharan P, Balzer JR, Anetakis K, Crammond DJ, Thirumala PD : Electrophysiology of extraocular cranial nerves: oculomotor, trochlear, and abducens nerve. J Clin Neurophysiol 35 : 11-15, 2018

4. Kaspera W, Adamczyk P, Ślaska-Kaspera A, Ładziński P : Usefulness of intraoperative monitoring of oculomotor and abducens nerves during surgical treatment of the cavernous sinus meningiomas. Adv Med Sci $60: 25-30,2015$

5. Kassam AB, Prevedello DM, Carrau RL, Snyderman CH, Thomas A, Gardner $\mathrm{P}$, et al. : Endoscopic endonasal skull base surgery: analysis of complications in the authors' initial 800 patients. J Neurosurg 114 : 1544-1568, 2011

6. Kawaguchi M, Ohnishi H, Sakamoto T, Shimizu K, Touho H, Monobe $\mathrm{T}$, et al. : Intraoperative electrophysiologic monitoring of cranial motor nerves in skull base surgery. Surg Neurol 43 : 177-181, 1995

7. Kawamata T, Ishii N, Amano K, Namioka T, Hori T, Okada Y : A novel simple real-time electrooculographic monitoring system during transsphenoidal surgeries to prevent postoperative extraocular motor nerve dysfunction. Neurosurg Rev 36 : 371-376, 2013

8. López JR : Neurophysiologic intraoperative monitoring of the oculomotor, trochlear, and abducens nerves. J Clin Neurophysiol 28 : 543550,2011

9. Schlake HP, Goldbrunner R, Siebert M, Behr R, Roosen K : Intra-operative electromyographic monitoring of extra-ocular motor nerves (Nn. III, VI) in skull base surgery. Acta Neurochir (Wien) $143:$ 251-261, 2001

10. Sekiya T, Hatayama $T$, Iwabuchi $T$, Maeda $S$ : Intraoperative recordings of evoked extraocular muscle activities to monitor ocular motor nerve function. Neurosurgery 32 : 227-235; discussion 235, 1993

11. Sheshadri V, Bharadwaj S, Chandramouli BA : Intra-operative electrooculographic monitoring to prevent post-operative extraocular motor nerve dysfunction during skull base surgeries. Indian J Anaesth 60 : 560-565, 2016

12. Singh $\mathrm{H}$, Vogel RW, Lober RM, Doan AT, Matsumoto Cl, Kenning TJ, et al. : Intraoperative neurophysiological monitoring for endoscopic endonasal approaches to the skull base: a technical guide. Scientifica (Cairo) 2016 : 1751245, 2016

13. Thirumala PD, Mohanraj SK, Habeych M, Wichman K, Chang YF, Gardner $P$, et al. : Value of free-run electromyographic monitoring of extraocular cranial nerves during expanded endonasal surgery (EES) of the skull base. J Neurol Surg Rep 74 : 43-50, 2013

14. Zhou Q, Zhang $\mathrm{M}$, Jiang $\mathrm{Y}$ : Intraoperative oculomotor nerve monitoring predicts outcome following clipping of posterior communicating artery aneurysms. J Clin Neurosci 19 : 706-711, 2012 